blieb oftmals offen. Dieses begriffliche Potpourri ist mit Sicherheit zum Teil der sozialwissenschaftliche Diskussion der letzten Jahre um Wissen geschuldet. Statt, wie von den Veranstaltern intendiert, gemeinsame Perspektiven zwischen Politikwissenschaft, Soziologie und Science and Technology Studies auszuloten, könnten aber die Perspektiven klassischer sozialwissenschaftlicher und besonders wissenssoziologischer Theoriebildung ausreichen, um aktuelle Forschungsprojekte hinreichend zu beschreiben. Dementsprechend wären die Vorträge Straßheims, Voss', Palfners und Niesytos als Beispiele für das anfangs skizzierte Foucaultsch inspirierte Forschungsinteresse zu verstehen. Bei den Beiträgen von Petersen, Böschen, Beck, Löber, Fischer und Fröhlich handelt es sich um Projekte, die sich für die sozialen bzw. sozialwissenschaftlichen Konsequenzen interessieren, die sich ergeben, wenn soziale Subjekte kontextspezifisch extern konstruierte Wissensbestände interpretieren. Natürlich überlappen sich die analytischen Perspektiven. Jedoch kristallisierte sich immer ein eindeutiges Erkenntnisinteresse heraus. Nicht bezweifelt werden soll, dass die vorgestellten Forschungsprojekte auf höherer Detailebene mit weitergehenden analytischen Kategorien arbeiten. Folglich zeigte der Workshop, dass politikwissenschaftliche Perspektiven bei der Erforschung des Verhältnisses von Politik und Wissen eher als Residualkategorie denn als Anleitung für Projekte dienen. Diese Beobachtung verwundert, handelt es sich bei dem Organisator des Workshops doch um den Arbeitskreis Politik, Wissenschaft und Technik der Deutschen Vereinigung für Politikwissenschaft.

$\langle\gg$

\section{„Kultur” als Herausforderung für Nachhaltigkeitsforschung}

Bericht vom 7. Workshop des „Forums

on Sustainable Technological Development in a Globalising World"

Berlin, 9.-12. Juni 2010

\section{von Barbara Muraca, Universität Greifswald, und Verena Holz, Universität Lüneburg}

Die Globalisierung bringt Herausforderungen für die Zukunftsgestaltung von Gesellschaften mit sich. Diese Herausforderungen werden auch in ihren kulturellen Zusammenhängen diskutiert. Während einige Ökonomen heute dafür plädieren, soziale und kulturelle Interessen der Individuen in die Indizierung von Entwicklung und Wachstum mit aufzunehmen, konzipieren Künstler und $\mathrm{Ku}-$ ratoren Ausstellungen, die sich mit den kulturellen Folgen des Klimawandels auseinandersetzen. Auch in der Netzwelt haben die Wechselbeziehungen nachhaltiger Entwicklung und Kultur ihre Plattformen gefunden: Während das Goethe-Institut etwa ein Portal „Klimawandel und Kultur“ und sogar einen entsprechenden Facebook-Account unterhält, debattieren weltweit User in zahlreichen eigens dafür eingerichteten Blogs über „Culture and Sustainability“. Diese heterogenen Beispiele der Diskurse und Aktivitäten unterschiedlichster Akteure bilden ein Interesse hinsichtlich globaler Entwicklungspolitik und deren Strategien ab, das zunehmend ernstgenommen und auch wissenschaftlich diskutiert wird.

Der wissenschaftliche Nachhaltigkeitsdiskurs ist in seinem Kern transdisziplinär: Er verortet sich an der Schnittstelle zwischen interdisziplinärer (theoretischer wie auch angewandter) wissenschaftlicher Forschung, politischer Zielsetzung und politischen Entscheidungsprozessen sowie gesellschaftlicher Gestaltung, die eine möglichst breite Partizipation aller Beteiligten fordert und braucht. Die kulturelle Dimension nachhaltiger Entwicklung nicht weiterhin als „Beiwerk nachhaltiger Entwicklung“ zu betrachten, sondern als Struktur gebende modellierende Instanz in ihren Funktionen zu erforschen und wahrzunehmen, ist zu einer Herausforderung zeitgenössischer Wissenschaft geworden. 


\section{Kulturelle Dimensionen von Nachhaltigkeit}

Dieser Herausforderung haben sich 33 inter- und transdisziplinär arbeitende WissenschaftlerInnen aus 12 Ländern gestellt, die auf dem Workshop „Sustainability 2010: The Cultural Dimension" zusammenkamen. Im Zentrum stand die Frage nach den Berührungspunkten zwischen nachhaltiger Entwicklung und Kultur aus der Perspektive der jeweiligen unterschiedlichen Disziplinen und Kontexte. Der Workshop wurde vom Karlsruher Institut für Technologie (KIT/ ITAS) in Zusammenarbeit mit dem Florida Institute of Technology, College of Sciences, der Budapest University of Technology and Economics, Department of Management and Corporate Economics sowie der Leibniz-Sozietät der Wissenschaften zu Berlin veranstaltet.

Technologische Innovationen seien keine bloßen Instrumente menschlichen Handelns, wie Imre Hronszky in seiner Begrüßungsrede betonte. Vielmehr sei Technologie ein Medium, durch das Menschen miteinander vernetzt würden, und als solches wertbehaftet, symbolisch und sinnstiftend. Die steigende Komplexität der Systeme, in denen wir uns bewegen, fordere außerdem eine nahezu unendliche Anzahl an Betrachtungsperspektiven, in denen Kultur- und Kontextbezogenheit eine unverzichtbare Bereicherung darstellten. Umso mehr treffe dies auf nachhaltige Entwicklung zu, die, so Gordon Nelson, auf eine langfristige Zeitskala ausgerichtet sei und daher über einzelne nachhaltige Technologielösungen sowie über ökonomische und politische Zyklen hinaus gehen müsse. Da das, was wir wertschätzten, ein Ergebnis kultureller Prägung sei, bräuchten wir für die langfristige Sicherung eines Nachhaltigkeitspfades einen kulturellen Wandel im Sinne nachhaltiger Entwicklung. Die Kernfrage, die sich uns stelle, sei ob und wie dieser Wandel überhaupt zustande kommen kann.

Um die kulturellen Voraussetzungen, Bedingungen, Kontexte und Widerstände nachhaltiger Entwicklung zu systematisieren, wurden im Vorfeld drei thematische Schwerpunkte gesetzt, die den Workshop strukturierten: „Meanings of Sustainable Development", „Cultural Diversity and Sustainability - Implicit Norms and Values“ und „Sustainability - Political and Educational Implications“.

\section{Bedeutungen nachhaltiger Entwicklung}

Angesichts zahlreicher, teilweise vom Forschungskontext und politischen Rahmenbedingungen abhängiger Definitionen nachhaltiger Entwicklung wurden zunächst kulturelle Fragestellungen und Perspektiven in ihrem Verhältnis zur nachhaltigen Entwicklung diskutiert. Kultur als Dimension stand dabei im Spannungsfeld von Natur, Gesellschaft/en, Werten und Wertesystemen. Weitgehend einig waren sich die Eingangsredner Nelson Goodman, Medardo Uribe und Imre Hronsky in ihrem Grundverständnis von Kultur als strukturgebendem Ensemble von geteilten Haltungen, Zielen, Werten, Praktiken und Methoden. Ágnes Fésüs und Imre Hronszky wiesen dabei auf die Stabilisierungsfunktionen von Kultur als gesellschaftlichem Rahmen hin und hoben hervor, dass dieser ein Set von nichtreflektierten, impliziten Prinzipien enthalte, deren Reflexion jedoch im Kontext nachhaltiger Entwicklung unerlässlich sei.

Medardo Uribe führte ein Verständnis von Kultur als „Befreiung“ des Menschen von der ihn in seine Grenzen zwingenden Natur an - ein Verständnis, das ethymologisch seit der Antike im europäischen Raum Bestand hat, ideell bereits in der biblischen Schöpfungsgeschichte verankert ist und in teleologischen Vorstößen kulminiert, die den Menschen und seine Kultur als Endzweck der Natur betrachten. Mit Blick auf die Gesellschaft könne Kultur als übergeordnete, Struktur gebende und organisierende Entität und als sozialer Motor verstanden werden. In diesem Rahmen ließen sich auch Entwicklungsparadigmen interpretieren. Die klassischen Modernisierungstheorien etwa spiegelten als Teil von Kultur vorherrschende Deutungsmuster einer bestimmten Sichtweise wider, so Uribe. Auch wenn viele der Ziele und Ideale dieser Theorien einer Nachhaltigen Entwicklung entgegenstünden, sei nicht zu verkennen, dass nachhaltige Entwicklung im Kontext solcher konventioneller Entwicklungsparadigmen analysiert werden müsse.

Auch Nelson Goodman beleuchtete das Verhältnis von Wachstum und Kultur. Die Ent- 
wicklung des US-Bundesstaates Florida zeige, dass die Indikatoren für die Beschreibung einer „Wachstumskultur“ künftig andere sein müssten. Von einem an Fortschritt orientiertem linearen Denken, in dem Technologien als Mittel zur Verwirklichung von Zielen dienten, müsse heute zu einem selbstkritischen holistischen Denken übergegangen werden, das ethische und axiologische Fragen (Vorsorgeprinzip, Antizipationsund Adaptionsfähigkeit, Fragen der Orientierung etc.) berücksichtige.

In einer ähnlichen Richtung argumentierten auch Ursua Nicanor und Ignacio Ayesteran: Unterscheide man zwischen „benign problems“ und „wicked problems“", gehörten Fragen einer nachhaltigen Entwicklung aufgrund ihrer Komplexität und Irreversibilität zu der Kategorie der Wicked Problems. Traditionelle Lösungsverfahren, wie Zielsetzung und Feststellung der geeigneten Mittel, gingen an der Komplexität dieser Probleme vorbei. Benötigt würden neue Ansätze, die die raumzeitlichen Beziehungen in komplexen, dynamischen Systemen neu konzeptualisieren und nach dem Modell der post-normal-science operieren. Hierfür sei ein Schritt über die klassischen Grenzen wissenschaftlicher Forschung vonnöten hin zu einer Öffnung zu Axiologie, Ethik und dem gesellschaftspolitischen Diskurs. Komplexe sozioökologische Systeme bedürften innovativer transdisziplinärer Forschung.

Allerdings können Nachhaltigkeitsziele von der globalen und allgemeinen Ebene nicht einfach auf die lokale Ebene abgeleitet werden, da jede Kontextualisierung auch immer eine Interpretation und Adaption impliziere. Ein gutes Beispiel dieser Interpretationsprozesse lieferte Appukuttan Damodaran am Beispiel Indien. Neben den ,großen Traditionen“, die prägende kulturelle Orientierung auch hinsichtlich Nachhaltigkeitsfragen geben, existierten zahlreiche „kleine Traditionen“, die sich vor Ort als geprüfte Systeme des Umgangs mit natürlichen Ressourcen und unter den Mitgliedern der Gemeinschaft etabliert hätten. Diese zeigten eine höhere Permeabilität gegenüber exogenen Einflüssen und können - zum Teil - durch eine höhere Anpassungsfähigkeit Elemente westlicher Technologie und Kultur mit lokalen Traditionen in einen für die Gemeinschaft positiven Einklang bringen.
In der Diskussion wurde danach gefragt, inwieweit die im Nachhaltigkeitskonzept verankerten Ideen und Werte universell seien. An den Ansatz, eine Übersetzung in lokale Kontexte, Deutungssysteme und symbolische Dimensionen vorzunehmen, schloss sich die Frage an, wer diesen Transfer bewältigt und in wieweit eine solche Übersetzung gelingt. Ist es auch möglich, die Erzählungen und Interessen der Subalternen zu erkennen und einzubeziehen?

\section{Normen, Werte und Bildung}

Nachhaltige Entwicklung zielt als normatives Konzept auf einen gesellschaftlichen Werte- und Bewusstseinswandel - ist zugleich mit weltweit kulturell variierenden Wertesystemen konfrontiert. Zum Ausdruck kommt dies in den Kontroversen um die häufig zugrunde gelegten Werte „Demokratie“, „Menschenrechte“ und „Wachstum“. Die jeweiligen und unterschiedlichen Formulierungen der Leitidee einer nachhaltigen Entwicklung als normatives Ziel bzw. als regulatives Ideal wurden trotz der Festlegung von konkreten Handlungshinweisen bislang nicht umgesetzt. Wie Larissa Krainer durch Bezugnahme auf vielfältige Medienmaterialien zeigte, liegt dies u. a. an ihren Widersprüchen mit den herrschenden Wertesystemen in unserer Kultur. So herrsche eine hauptsächlich an technischen und ökonomischen Werten orientierte Kultur der Produktion und Konsumption, die Wettbewerb, Profitmaximierung, Beschleunigung und Innovationsdrang fordere. Da Werte und Wertesysteme auch innerhalb einer Kultur widersprüchlich seien, werde ein dialektischer Umgang mit Wertkonflikten benötigt.

Auch laut Robert Hauser steht das normative Konzept einer nachhaltigen Entwicklung in Widerspruch mit anderen geltenden normativen Konzepten und Wertvorstellungen, die zu interkulturellen Zusammenhängen quer lägen. Normen erzeugten Handlungsmuster, die für eine kulturelle Gemeinschaft identitätsstiftend wirkten: Wer sich nicht nach diesen Mustern verhalte, werde als Außenseiter wahrgenommen. Die Kategorie des Normativen werde unter dem „Axiologischen“ subsumiert und umfasse laut Hauser Bräuche, Sittennormen und Rechtsnormen (darunter Gesetze und Moralgesetze im en- 
geren Sinne), deren Verpflichtungsgrad jenseits von Aushandlungsprozessen stünden.

Vor diesem Hintergrund wurde im Folgenden die Rolle von Bildung für eine nachhaltige Entwicklung diskutiert. Wie Ryszard Janikowski und Andrzej Kiepas hervorhoben, könne man von einer Wechselwirkung zwischen Kultur, kulturell geprägten Wertsystemen und Bildung sprechen. Erst in den letzten Jahren habe sich in Polen das Konzept einer nachhaltigen Entwicklung etabliert, bei dem nicht nur ökologische und ökonomische Aspekte im Mittelpunkt stünden, sondern auch zunehmend soziale und kulturelle Aspekte. Kulturkapital - und dementsprechend Bildung spielten neben Sozial- und Humankapital eine zentrale Rolle im Nachhaltigkeitsdiskurs.

Werte und Wertesysteme hätten sich historisch entwickelt und etabliert. Wie János Szlavik und Füle Miklós zeigten, ist eine Rückkopplung an lokale Wertesysteme unverzichtbar für Modernisierungsprojekte im Sinne einer nachhaltigen Entwicklung. Historische Werte umfassten ästhetische und moralische Werte so wie auch die stratifizierte Erfahrung im Umgang mit Materialien und Dingen. Am Beispiel des Umgangs mit der Ressource Wasser zeigte Sharma Virender, dass die Berücksichtigung von lokalen Umwelt-, kulturellen und ökonomischen Bedingungen eine wesentliche Rolle spielt. Technische Lösungen bedürften einer Integration mit politischen Maßnahmen im Gesundheitssystem und ihr Erfolg sei von einer engen Zusammenarbeit mit den Partnern auf lokaler Ebene abhängig.

Im Zentrum einer weiteren Session standen Beispielprojekte einer Bildung für eine Nachhaltige Entwicklung, die kulturelle und ästhetische Fragestellungen explizit in ihre Konzeption mit einbeziehen. Oliver Parodi und Verena Holz hoben in diesem Zusammenhang einen anderen Teilbereich von Kultur hervor: Ästhetik, symbolische Ordnungen und Gestaltungsmöglichkeiten einer nachhaltigen Entwicklung wurden ins Verhältnis zum lernenden Individuum und seiner gesellschaftlichen Funktion gesetzt. Das Konzept der Bildung für eine nachhaltige Entwicklung sei nicht auf eine spezifische Zielgruppe ausgerichtet. Vielmehr sei es erklärtes Ziel, einen möglichst breiten Personenkreis in die Thematik einzubinden und Lernkontexte bereitzustellen, die alle Beteiligten dazu befähigten, aktiv an einer gemeinsamen Zukunftsgestaltung mit zu wirken.

\section{Nachhaltigkeit zwischen den Kulturen: Round-Table im Loebe-Haus}

Bestandteil des Workshops war auch eine RoundTable-Diskussion im Paul-Loebe-Haus des Deutschen Bundestags, die eingeleitet wurde mit einem Grußwort der SPD-Bundestagsabgeordneten Ulla Burchardt Sie hatte die politische Implementierung von Strategien "Nachhaltige Entwicklung“ als Vorsitzende der Querschnittsgruppe Nachhaltige Entwicklung (1998-2002) von Beginn an mit begleitet. Burchardt wies darauf hin, dass das Bewusstsein für die kulturelle Dimension nachhaltiger Entwicklung unerlässlich für ein Verständnis der eigentlichen Idee nachhaltiger Entwicklung sei - insbesondere, wenn es um die Einbettung in verschiedenartige Kontexte und Traditionen in unterschiedlichen Regionen dieser Welt gehe. In ihrer Funktion als Vorsitzende des Ausschusses für Bildung, Forschung und Technikfolgenabschätzung des Deutschen Bundestages hob Ulla Burchardt Bildung und Forschung als treibende Kräfte hinsichtlich Innovationen innerhalb des Wirtschafts- und Gesellschaftssystems hervor. Dabei bilde nachhaltige Entwicklung schon seit mehr als zehn Jahren die zentrale normative Orientierung. Insbesondere neu entwickelte Technologien für nachhaltige Entwicklung müssten ökonomische, soziale, ethische und kulturelle Gesichtspunkte in den Blick nehmen.

Die Diskussion fokussierte anschließend auf interkulturelle Aspekte einer nachhaltigen Entwicklung. Kulturelle Missverständnisse entstehen oft auf der Grundlage von selbstverständlichen und nicht weiter hinterfragten Annahmen über das eigene Verständnis von wesentlichen Kategorien wie Zeit, Raum, Relationen und Wandel. So hob z. B. Silja Graupe in ihrem Diskussionsbeitrag hervor, dass beim chinesischen und japanischen Buddhismus das Grundverständnis der Welt nicht aus „Dingen“ selbst, sondern vielmehr aus Relationen und Prozessen ausgehe. Vor diesem Hintergrund seien Begriffe wie „Bewahren und Aufbewahren" irreführend. Auch finde sich in Indien ein anderes Verständnis von Raum und Zeit: Zeit sei aus einer Reinkarnationsper- 
spektive unendlich und die Pflichten gegenüber künftigen Generationen gestalteten sich in einem ganz anderen Zusammenhang (Appukuttan Damodaran). In diesem Sinne meinte auch Medardo Tapia Uribe, dass nachhaltige Entwicklung als Prozess und nicht nur als Ziel zu verstehen sei. Dabei solle die Zivilgesellschaft mit ihren vielfältigen und komplexen Strukturen die zentrale Rolle spielen. Eine breite Partizipation aller Stimmen sei fundamental, wie er am Beispiel des Widerstandes der lokalen indigenen Bevölkerung in Mexiko gegen bestimmte Formen von Entwicklung und Wachstum zeigte. Caroline Robertson-von Trotha hob schließlich hervor, dass der kulturelle Pluralismus auf lokaler Ebene eine unverzichtbare Ressource sei, mit der noch nicht richtig umgegangen werde (,Kultur des kulturellen Pluralismus").

\section{Abschließende Bemerkungen}

Instruktiv war die Tatsache, dass sich die Teilnehmenden nicht von Beginn an auf eine Definition von Kultur festlegten. Insofern war im Sinne der Inter- und Transdisziplinarität ein aufschlussreiches Verfahren der Terminologisierung von „Kultur“ und „nachhaltiger Entwicklung“ zu beobachten. Alle Beteiligten waren sich einig, dass in einem nächsten Schritt die Erarbeitung einer methodologisch angemessenen Erfassung des Verhältnisses nachhaltiger Entwicklung und der kulturellen Dimension folgen solle. Die Erkenntnis, dass Nachhaltigkeit nur aus einer transdisziplinären Perspektive fokussiert werden kann, die nicht nur die Zusammenarbeit verschiedener wissenschaftlicher Disziplinen und Praxis impliziert, sondern vielmehr ein Umdenken im Verständnis von Wissenschaft selbst erfordert, stellt die größte Herausforderung dar. Diese kann nur in Form eines gemeinsamen Austauschs bewältigt werden, gleichzeitig zeigt sie aber auch die Notwendigkeit der wissenschaftlichen Ausgestaltung des Forschungsgegenstands.

\langle\rangle

\section{Perspektiven der sozialwissen- schaftlichen Technik- und Inno- vationsforschung}

Klausurtagung der Sektion Wissenschafts- und Technikforschung der Deutschen Gesellschaft für Soziologie

Stuttgart, 8.-9. Juli 2010

\section{von Ulrich Dolata, Jürgen Schrape und Stephanie Schulz, Universität Stuttgart}

Wohin könnte und sollte sich die sozialwissenschaftliche Technik- und Innovationsforschung in den kommenden Jahren bewegen? Welche theoretischen Themen und Ansätze, welche empirischen Untersuchungsgegenstände und welche methodischen Fragen sind besonders viel versprechend und zukunftsträchtig? Welche Impulse aus der internationalen Diskussion sollten in der deutschsprachigen Technik- und Innovationsforschung stärker aufgegriffen werden? Und welche Impulse könnte umgekehrt der deutschsprachige Diskurs stärker in die internationale Diskussion hineintragen?

Um diese Fragen kreisten die Diskussionen auf einer vom Lehrstuhl für Organisations- und Innovationssoziologie ausgerichteten Klausurtagung zu den „Perspektiven der sozialwissenschaftlichen Technik- und Innovationsforschung“, die im Juli 2010 an der Universität Stuttgart stattfand. Die Tagung brachte 35 namhafte Wissenschaftlerinnen und Wissenschaftler zu einem intensiven Meinungsaustausch über den aktuellen Stand der Forschung und die künftigen Forschungsschwerpunkte des Fachs zusammen.

Nach einer ersten allgemeinen Debatte um die kontrovers diskutierte Frage, inwieweit der Innovationsbegriff auf soziale Zusammenhänge (soziale Innovationen) ausgeweitet werden sollte (Impulsstatements: Werner Rammert, TU Berlin, und Armin Grunwald, ITAS), wurden fünf Themenbereiche erörtert, in die jeweils durch kurze Impulsstatements eingeführt wurde:

- Veränderungen von Arbeitsprozessen und Alltagszusammenhängen durch neue Technologien (insbesondere durch das Internet), durch intelligente Infrastrukturtechnologien 